\title{
Modeling Influenza Viral Dynamics in Tissue
}

\author{
Catherine Beauchemin ${ }^{1, \star}$, Stephanie Forrest ${ }^{2}$, and Frederick T. Koster ${ }^{3}$ \\ ${ }^{1}$ Adaptive Computation Lab., University of New Mexico, Albuquerque, NM \\ cbeau@cs.unm.edu \\ ${ }^{2}$ Dept. of Computer Science, University of New Mexico, Albuquerque, NM \\ ${ }^{3}$ Lovelace Respiratory Research Institute, Albuquerque, NM
}

\begin{abstract}
Predicting the virulence of new Influenza strains is an important problem. The solution to this problem will likely require a combination of in vitro and in silico tools that are used iteratively. We describe the agent-based modeling component of this program and report preliminary results from both the in vitro and in silico experiments.
\end{abstract}

\section{Introduction}

Influenza, in humans, is caused by a virus that attacks mainly the upper respiratory tract, the nose, throat and bronchi and rarely also the lungs. According to the World Health Organization (WHO), the annual influenza epidemics affect from $5 \%$ to $15 \%$ of the population and are thought to result in 3-5 million cases of severe illness and 250,000 to 500,000 deaths every year around the world [1]. The rapid spread of H5N1 avian influenza among wild and domestic fowl and isolated fatal human cases of H5N1 in Eurasia since 1997, has re-awakened interest in the pathogenesis and transmission of influenza A infections [2]. The most feared strain would mimic the 1918 strain which combined high transmissibility with high mortality 34]. Virulence of influenza viruses is highly variable, defined by lethality and person-to-person transmission, but the causes of this variability are incompletely understood. The early events of influenza replication in airway tissue, particularly the type and location of early infected cells, likely determine the outcome of the infection. Rate of airway tissue spread is controlled by efficiency of viral entry and exit from cells, variable intracellular interferon activation modulated by the viral NS-1 protein, and by an array of extracellular innate defenses. Although molecular biology has provided a detailed understanding of the replication cycle in immortalized cells, influenza replication in intact tissue among phenotypically diverse epithelial cells of the human respiratory tract remains poorly understood. We are missing a quantitative accounting of kinetics in the human airway and an explanation for how one strain, but not a closely related strain, can initiate person-to-person transmission.

Although the viral structure and composition of influenza are known, and even some dynamical data regarding the viral and antibody titers over the course of the infection [5|67, key information such as the shape and magnitude of the viral burst, the length of the viral replication cycle (time between entry of the

^ Corresponding author. 
first virus and release of the first produced virus), and the proportion of productively infectious virions, is either uncorroborated, unknown, or known with poor precision. This makes modeling influenza from data available in the literature a near impossibility, and it points to the need for generating experimental data aimed directly at the needs of both computational and mathematical models.

This paper describes the computer modeling side of a project that is integrating in vitro experiments with computer modeling to address this problem. We are focusing on the early dynamics of influenza infection in a human airway epithelial cell monolayer using both in vitro and computer models. The in vitro model uses primary human differentiated lung epithelial cells grown in an air-liquid interface (ALI) culture to document the kinetics of influenza spread in tissue. The computer model consists of an agent-based model (ABM) implementation of the in vitro system. Its architecture is modular so that more details can be added whenever data from the in vitro system justifies it. Here, we will describe the implementation of the computer model and report some initial simulation results.

To our knowledge, only four mathematical models for influenza dynamics have ever been proposed. The first and oldest one is from 1976 and consists of a very basic compartmental model for influenza in experimentally infected mice [8]. After a gap of 18 years, Bocharov et al. proposed an exhaustive ordinary differential equation model based on the basic viral infection model but extended to include 12 different cell populations described by 60 parameters 9 . More recently, one of us co-authored a paper presenting another ordinary differential equation model with very slight modifications from the basic viral infection model [10] and a second paper presenting a simple ABM for influenza [11. All of these models either perform poorly when compared to experimental data or are too simplistic to capture the dynamics of interest in influenza.

\section{$2 \quad$ Agent-Based Modeling}

The spatial distribution of agents is an important and often neglected aspect of influenza dynamics. We capture spatial dynamics through the use of an agentbased model (also known as an individual-based) cellular automata style model. Each epithelial cell in the monolayer is represented explicitly, and a computer program encodes the cell's behavior and rules for interacting with other cells and its environment. The cells live on a hexagonal lattice and interact locally with other cells and virions in their neighborhood following a set of predefined rules. Thus, the behavior of the low-level entities is pre-specified, and the simulation is run to observe high-level behaviors (e.g. to determine an epidemic threshold). This style of modeling emphasizes local interactions, and those interactions in turn give rise to the large-scale complex dynamics of interest.

This modeling approach can be more detailed than other approaches. The programs can directly incorporate biological knowledge or hypotheses about low-level components. Data from multiple experiments can be combined into a single simulation, to test for consistency across experiments or to identify gaps 
in our knowledge. Through its functional specifications of cell behavior, our can potentially bridge the current gap between intracellular descriptions and infection dynamics models. Similar approaches have been used to model a variety of host-pathogen systems ranging from general immune system simulation platforms [12 13 14 15 16] to models of specific diseases including tuberculosis [17,18],

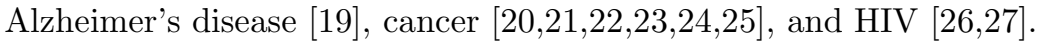

The spatially explicit agent-based approach is an appropriate method for this project. The ALI is a complex biological system in which many different defenses (e.g. mucus, cytokines) interact and biologically relevant values cannot always be measured directly. In addition, recent high-profile publications have demonstrated that entry of avian and human-adapted influenza viruses into different airway epithelial cells depends on the cell receptor which in turn is dependent on cell type and location in the airway 28,29]. Our modeling approach will facilitate the exploration of spatially heterogeneous populations of cells.

\section{Influenza Model}

Our current model is extremely simple. We plan to gradually add more detail, ensuring at each step that the additions are justified by our experimental data. Here, we describe the model as it is currently implemented.

We are modeling influenza dynamics on an epithelial cell monolayer in vitro. The monolayer is represented as a two-dimensional hexagonal lattice where each site represents one epithelial cell. The spread of the infection is modeled by including virions. Rather than treat each virion explicitly, the model instead considers the concentration of virions by associating a continuous real-valued variable with each lattice site, which stores the local concentration of virions at that site. These local concentrations are then allowed to change, following a discretized version of the diffusion equation with a production term. The rules governing epithelial cell and virion concentration dynamics are described below.

\subsection{Epithelial Cell Dynamics}

The epithelial cells can be found in any of the four states shown in Fig.1, namely healthy, containing, secreting, and dead. For simplicity, we assume that there is no cell division or differentiation over the course of the infection. The parameters responsible for the transition between these states are as follows.

Infection of Epithelial Cells by Virions $(\boldsymbol{k})$ : Each site keeps track of the number of virions local to the site, $V_{m, n}$. But while there are $V_{m, n}$ virions at site $(m, n)$ at a given time step, depending on the length of a time step, not all of these virions necessarily come in contact with the cell, and some may contact it more than once. Alternatively, a particular strain of virions may not be as successful at binding the cell's receptors and being absorbed by the cell. To reflect this reality, we introduce the parameter $k$ which gives the probability per hour per virion 


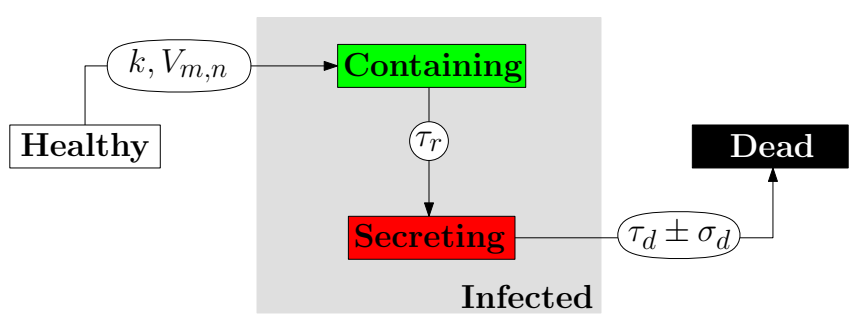

Fig. 1. The agent-based model's four states for epithelial cells, (Healthy, Containing, Secreting, and Dead), and the parameters responsible for controlling the transitions between these states

that a healthy cell will become infected (enter the containing stage). In other words, $k \times V_{m, n}$ gives the probability that the healthy cell located at site $(m, n)$ will become infected over the course of an hour. In order to fit experimental data, we set the rate of infection of cells per virions in our model to $k=8$ per virion at that site per hour.

Duration of the Viral Replication Cycle $\left(\tau_{r}\right)$ : This variable represents the time that elapses between entry of the first successful virion and release of the first virion produced by the infected cell. From the experiments, we found this to be about $7 \mathrm{~h}$, and hence we set $\tau_{r}=7 \mathrm{~h}$ in the ABM.

Lifespan of Infectious Cells $\left(\tau_{\boldsymbol{d}} \pm \boldsymbol{\sigma}_{\boldsymbol{d}}\right)$ : Once infected (containing), a cell typically lives $24 \mathrm{~h}-36 \mathrm{~h}$ (from experimental observations). Given that the replication cycle lasts $\tau_{r}=7 \mathrm{~h}$, this means that once it starts secreting virions, an infectious cell typically lives $17 \mathrm{~h}-29 \mathrm{~h}$ or about $23 \pm 6 \mathrm{~h}$. Thus, we set the lifespan of each infected cell individually by picking it randomly from a Gaussian distribution of mean $\tau_{d}=23 \mathrm{~h}$ and standard deviation $\sigma_{d}=6 \mathrm{~h}$. In our ABM, cell death is taken to mean the time at which cells cease to produce virions. Note that in vitro, a cell undergoing apoptosis will eventually detach from the monolayer and will be replaced by a differentiating basal cell. For the moment, we neglect these processes and reduce their impact by fitting our ABM to experimental results over no more than the first $25 \mathrm{~h}$ after virion deposition.

\subsection{Virion Dynamics}

As mentioned earlier, virions are not represented explicitly. Instead, we track the concentration of virions stored as a real-valued continuous variable at each site of the lattice. The diffusion of virions is then modeled using a finite difference approximation to the diffusion equation. The continuous diffusion equation of the concentration of virions, $V$, is described by

$$
\frac{\partial V}{\partial t}=D_{V} \nabla^{2} V
$$


where $V$ is the concentration of virions, $\nabla^{2}$ is the Laplacian, and $D_{V}$ is the diffusion coefficient. The simulation is run on a hexagonal grid. The geometry of the grid and the base vectors we chose are illustrated in Fig.2

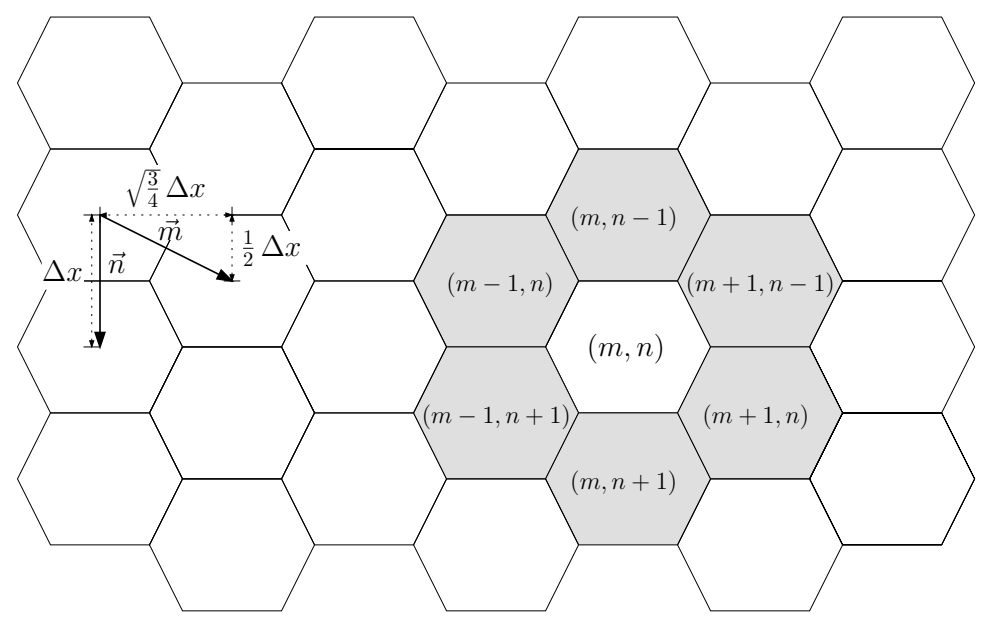

Fig. 2. Geometry of agent-based model's hexagonal grid. The honeycomb neighborhood is identified in gray, and the base vectors $\boldsymbol{m}$ and $\boldsymbol{n}$ are shown and expressed as a function of $\Delta x$, the grid spacing which is the mean diameter of an epithelial cell.

We can express (1) as a difference equation in the hexagonal coordinates $(m, n)$ as a function of the 6 honeycomb neighbors as

$$
\frac{V_{m, n}^{t+1}-V_{m, n}^{t}}{\Delta t}=\frac{4 D_{V}}{(\Delta x)^{2}}\left[-V_{m, n}^{t}+\frac{1}{6} \sum_{\text {nei }} V_{\text {nei }}^{t}\right] \text {, }
$$

such that $V_{m, n}^{t+1}$ at time $t+1$ as a function of $V_{m, n}^{t}$ and its 6 honeycomb neighbors $V_{\text {nei }}^{t}$ at time $t$ is given by

$$
V_{m, n}^{t+1}=\left(1-\frac{4 D_{V} \Delta t}{(\Delta x)^{2}}\right) V_{m, n}^{t}+\frac{2 D_{V} \Delta t}{3(\Delta x)^{2}} \sum_{\text {nei }} V_{\text {nei }}^{t},
$$

where $\sum_{\text {nei }} V_{\text {nei }}^{t}$ is the sum of the virion concentration at all 6 honeycomb neighbors at time $t$.

Because we want to simulate the infection dynamics in an experimental well, we want the diffusion to obey reflective boundary conditions along the edge of the well. Namely, we want $\frac{\partial V}{\partial j}=0$ at a boundary where $j$ is the direction perpendicular to the boundary. It can be shown that for such a case, (3) becomes

$$
V_{m, n}^{t+1}=\left(1-N_{\text {nei }} \frac{2 D_{V} \Delta t}{3(\Delta x)^{2}}\right) V_{m, n}^{t}+\frac{2 D_{V} \Delta t}{3(\Delta x)^{2}} \sum_{N_{\text {nei }}} V_{N_{\text {nei }}}^{t}
$$


where $N_{\text {nei }}$ is the number of neighbors a cell really has. Note that for $N_{\text {nei }}=6$, (4) reduces to (3).

The virion-related parameters $D_{V}, \Delta x, \Delta t$ in (4), and the release rate of virions, $g_{V}$, have been set as follows.

Diffusion Rate of Virions $\left(\boldsymbol{D}_{\boldsymbol{V}}\right)$ : The diffusion rate or diffusion coefficient for virions, $D_{V}$, measures how fast virions spread: the larger $D_{V}$, the faster virions will spread to neighboring sites and then to the entire grid. One way to determine $D_{V}$ from experimental results is to take a measure of the "patchiness" of the infection, i.e. the tendency of infected cells to be found in batches. The autocorrelation function offers a good measure of patchiness. Hence, we calibrated $D_{V}$ by visually matching our simulation to the experimental autocorrelation. We started with $D_{V}=3.18 \times 10^{-12} \mathrm{~m}^{2} / \mathrm{s}$ which is the diffusion rate predicted by the Stokes-Einstein relation for influenza virions diffusing in plasma at body temperature. Ultimately, we found that $D_{V}=3.18 \times 10^{-15} \mathrm{~m}^{2} / \mathrm{s}$, a value 1,000 fold greater than the Stokes-Einstein diffusion, yielded the best agreement to the experimental autocorrelation. This is illustrated in Fig. 3 where the experimental autocorrelation is plotted against simulation results for different values of $D_{V}$.

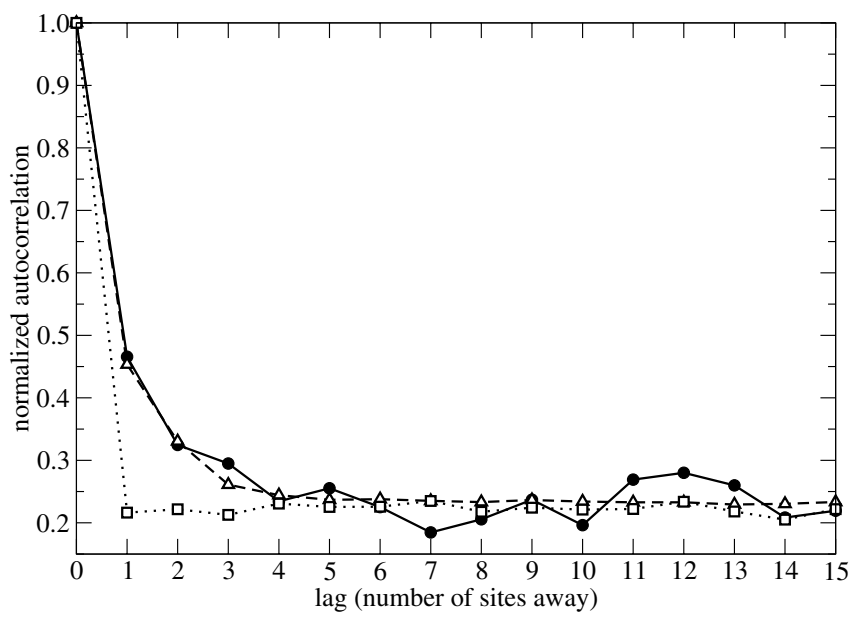

Fig. 3. Autocorrelation at $24 \mathrm{~h}$ post-harvest for the experiments (full line, full circles) compared against the autocorrelation produced by the simulation when using a diffusion coefficient of $D_{V}=3.18 \times 10^{-12} \mathrm{~m}^{2} / \mathrm{s}$ (dotted line, empty squares), and $D_{V}=3.18 \times 10^{-15} \mathrm{~m}^{2} / \mathrm{s}$ (dashed line, empty triangles). All parameters are as in Table 1 except for the $D_{V}=3.18 \times 10^{-12} \mathrm{~m}^{2} / \mathrm{s}$ simulation where $k$ was set to 4 per virions per hour to preserve the same fraction of cells infected at $24 \mathrm{~h}$ post-harvest. The autocorrelation have been "normalized" to be one for a lag of zero.

Grid Spacing or Diameter of Epithelial Cells $(\boldsymbol{\Delta x})$ : The diameter of epithelial cells was estimated from "en face" and cross-section pictures of the experimental monolayer. The average epithelial cell diameter was found to be about $11 \pm 1 \mu \mathrm{m}$. We use $\Delta x=11 \mu \mathrm{m}$. 
Duration of a Time Step $(\Delta t)$ : The stability criterion for the finite difference approximation to the diffusion equation presented in (4) requires that

$$
\Delta t \leq \frac{(\Delta x)^{2}}{4 D_{V}},
$$

which is a more stringent requirement for larger values of $D_{V}$ or smaller values of $\Delta x$. We use $\Delta x=11 \mu \mathrm{m}$ which is the diameter of lung epithelial cells, and $D_{V}=3.18 \times 10^{-15} \mathrm{~m}^{2} \cdot \mathrm{s}^{-1}$ such that in order to satisfy the stability criterion, we need $\Delta t \leq 2.6 \mathrm{~h}$. We found that setting $\Delta t=2 \mathrm{~min}$ satisfies the stability criterion of the diffusion equation and accurately captures the behaviour of the system.

Virion Release Rate $\left(\boldsymbol{g}_{\boldsymbol{V}}\right)$ : As seen above, $\tau_{r}=7 \mathrm{~h}$ after becoming infected, an epithelial cell will start secreting virions. In the model, secreting cells release virions at a constant rate until the cell is considered "dead", at which time secretion is instantaneously stopped. This "shape" for the viral burst was chosen arbitrarily as very little is known about the shape, duration, and magnitude of the viral burst. We found that setting the release rate of virions by secreting cells to $g_{V}=0.05$ virions per hour per secreting cell in our ABM yields a good fit of the simulation to the experimental data.

\subsection{Setting Up the Model}

The infection of the epithelial cell monolayer with influenza virions in our in vitro experiments proceeds as follows. An inoculum containing 50, 000 competent virions (or 50,000 plaque forming unit or pfu) is deposited evenly on the cell monolayer. The solution is left there for one hour to permit the infection of the cells and at time $t=0 \mathrm{~h}$, the inoculum is harvested with a pipette. At that time, not all the virions are removed: some are trapped in the mucus and get left behind.

To avoid having to model the initial experimental manipulations and the uncertainty in the viral removal, we start the ABM simulations at time $t=2 \mathrm{~h}$ post-harvest. At that time, a fraction of cells have been infected by the inoculum and a few virions have been left behind at harvest-time. To account for this fact, we define two more parameters, $V_{0}$ and $C_{0}$, which give the number of virions per cell and the fraction of cells in the containing stage at time $t=2 \mathrm{~h}$ postharvest, the initialization time of our simulations. In order to determine the number of virions per cell, we also defined $N_{\text {cells }}$, the number of epithelial cells in the experimental well. Parameters $N_{\text {cells }}, V_{0}$ and $C_{0}$ were set as follows.

Number of Epithelial Cells in the Experimental Well. $\left(N_{\text {cells }}\right)$ : We computed $N_{\text {cells }}$, the number of epithelial cells in the experimental well using the measured diameter of the epithelial cells, $\Delta x=11 \mu \mathrm{m}$, and the known area of the experimental well, $A_{\text {well }}=113 \mathrm{~mm}^{2}$. Assuming that the sum of the surface area 
of all the epithelial cells fully fills the well's area and that the surface area of each cell is roughly circular, such that $A_{\text {cell }}=\pi(\Delta x / 2)^{2}$, we can compute the number of epithelial cells in the experimental well

$$
\begin{gathered}
N_{\text {cells }}=\frac{A_{\text {well }}}{\pi(\Delta x / 2)^{2}} \\
=\frac{113 \mathrm{~mm}^{2}}{\pi(11 \mu \mathrm{m} / 2)^{2}} \\
\sim 1,200,000 \text { cells } .
\end{gathered}
$$

For our ABM, we found that setting the well radius of $R_{\text {well }}=160$ cells, which corresponds to about 93,000 simulated cells, is sufficient to accurately capture the behaviour of a full scale simulation.

Initial Number of Virions per Epithelial Cell $\left(\boldsymbol{V}_{\mathbf{0}}\right)$ : At time $t=2 \mathrm{~h}$ postharvest, the time at which we begin the simulation, $635 \pm 273$ virions were found on the monolayer. Hence, we can compute the number of virions per epithelial cell present on the monolayer at time $t=2 \mathrm{~h}$ post-harvest,

$$
\begin{gathered}
V_{0}=\frac{635 \text { virions }}{N_{\text {cells }}} \\
\sim 5.3 \times 10^{-4} \text { virions } / \text { cell },
\end{gathered}
$$

which corresponds to the number of virions per cell at initialization time.

Fraction of Cells Initially Infected $\left(\boldsymbol{C}_{\mathbf{0}}\right)$ : The parameter $C_{0}$ gives the fraction of cells which are initially set to the containing state. Those are the cells that were infected during incubation with the inoculum. Staining the ALI monolayer with viral antigen at $t=8 \mathrm{~h}$ post-harvest revealed that approximately $1.8 \%$ of the cells contained influenza protein, i.e. were producing virions. Hence, we set $C_{0}=0.018$ in the ABM such that $1.8 \%$ of cells are set to the containing stage at initialization time.

\section{Preliminary Results}

In its current implementation, the ABM has 11 parameters shown in Table1, A screenshot of the simulation grid is presented in Fig. 4, and Fig. 5 presents the dynamics of the various cell states and viral titer as a function of time against preliminary experimental data. We can see that the ABM provides a reasonable fit to the experimental data. 
Table 1. The 11 parameters used in the computer model, with a short description of their role and their default value. In the Source column, C stands for computed, $\mathrm{M}$ for measured experimentally, L for taken from the literature, and $\mathrm{F}$ for parameters adjusted in order to fit the model to the experiments.

\begin{tabular}{|c|c|c|c|}
\hline$\overline{\text { Symbol }}$ & Description & Value & Source \\
\hline \multicolumn{4}{|c|}{ Fixed Parameters } \\
\hline$R_{\text {well }}$ & radius of simulation well in \# cells & 160 cells & C (Sect.3.3) \\
\hline$\Delta t$ & duration of a time step & $2 \mathrm{~min} /$ time step & $\mathrm{C}$ (Sect. 3.2 ) \\
\hline$\Delta x$ & grid spacing (diameter of epithelial cells) & $11 \mu \mathrm{m}$ & M (Sect.3.2) \\
\hline$\tau_{r}$ & duration of the viral replication cycle & $7 \mathrm{~h}$ & L (Sect.3.2) \\
\hline$\tau_{d} \pm \sigma_{d}$ & infectious cell lifespan (mean $\pm \mathrm{SD})$ & $23 \pm 6 \mathrm{~h}$ & C (Sect.3.1) \\
\hline \multicolumn{4}{|c|}{ Adjusted Parameters } \\
\hline$C_{0}$ & fraction of cells initially infected & 0.018 & F (Sect.3.3) \\
\hline$V_{0}$ & initial dose of virions per cell & $5.3 \times 10^{-4}$ virions & F (Sect.3.3) \\
\hline$k$ & infection rate of cells by virions & $8 / \mathrm{h}$ & F (Sect.3.1) \\
\hline$g_{V}$ & rate of viral production per cell & $0.05 / \mathrm{h}$ & F (Sect.3.2) \\
\hline$D_{V}$ & diffusion rate of virions & $3.18 \times 10^{-15} \mathrm{~m}^{2} / \mathrm{s}$ & F (Sect. 3.2) \\
\hline
\end{tabular}

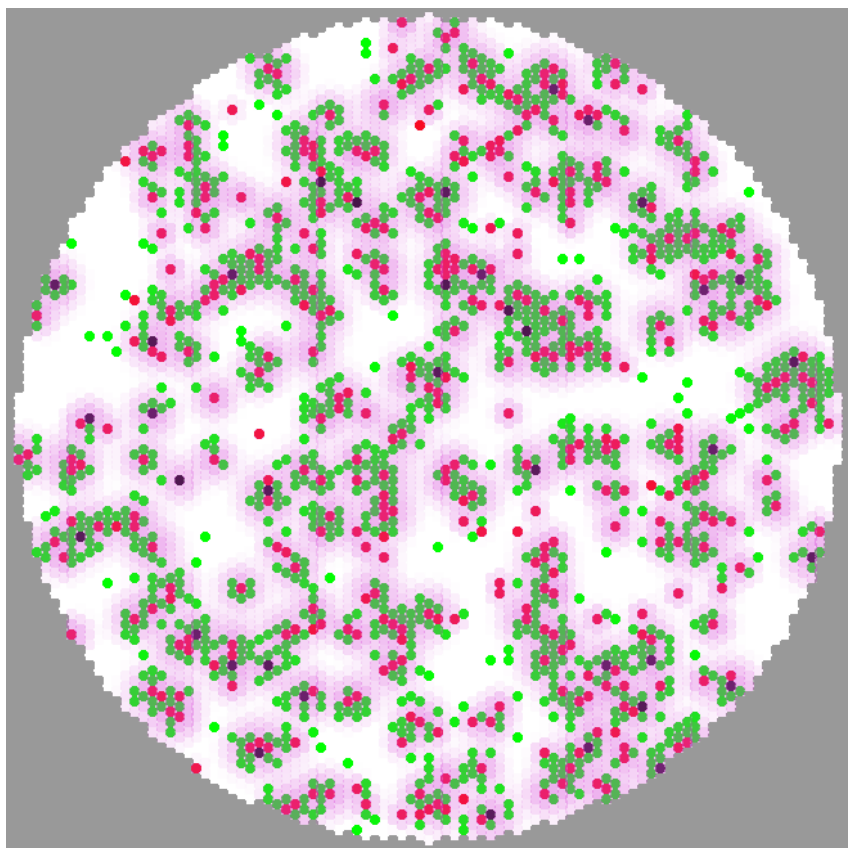

Fig. 4. Screenshot of the simulation taken at $18 \mathrm{~h}$ post-harvest for a simulated grid (well) containing 5,815 cells using the parameter values presented in Table 11 The cells are color-coded according to their states as in Fig. 1 with healthy cells in white, containing cells in green, secreting cells in red, and dead cells in black. The magenta overlay represents the concentration of virions at each site with more opaque magenta representing higher concentration of virions. 


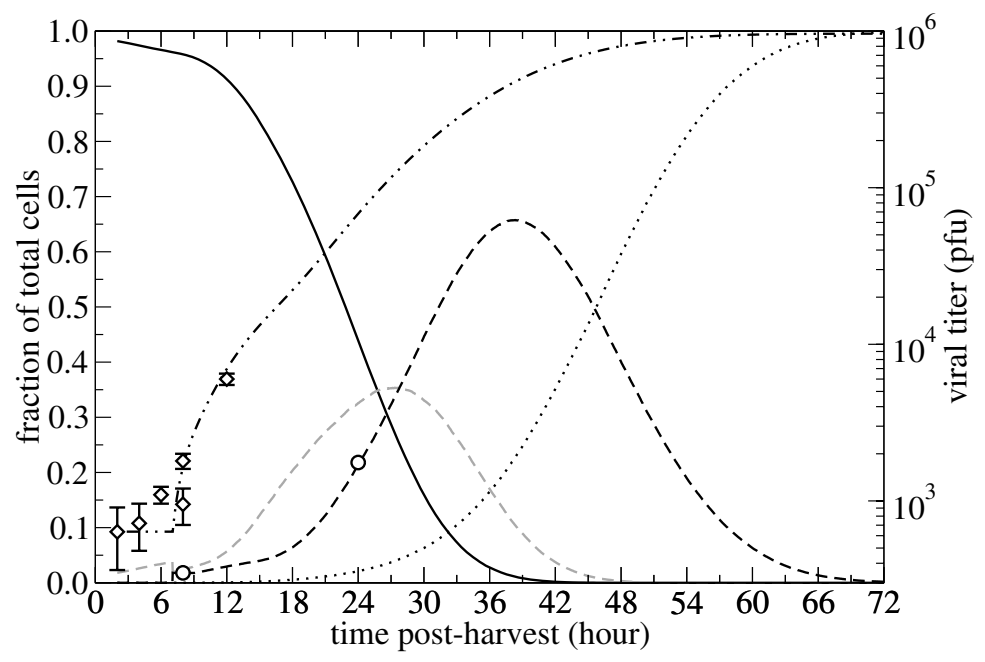

Fig. 5. Simulation results using the parameter set presented in Table 1 The lines represent the fraction of epithelial cells that are healthy (solid black), containing the virus (dashed grey), secreting the virus (dashed black), or dead (dotted black), as well as the number of competent virions (or pfu) on the right y-axis (dash-dot-dot black). The diamonds and the circles represent experimental data for the viral titer and the fraction of cells infected, respectively.

Note added in press: Recent experiments have revealed a highly variable dynamic range of the replication rate, but the basic structure of the model remains intact.

\section{Proposed Extensions}

As mentioned earlier, the current model is extremely simple, and we plan to gradually increase the level of detail.

One of the first improvements would be the inclusion of different cell types. The epithelial cells that make up the simulation grid are assumed to be a homogeneous population of cells, with no distinction, for example, between ciliated and Clara cells. We plan to add more cell types; each cell type would have the same four states illustrated in Fig.1, and the transitions between those states would still be dictated by the same processes, but the value of the parameters controlling these processes would differ from one cell type to another and from one virus strain to another. With such a model, we could, for example, explore differences in the spread of the infection on a sample constituted of $90 \%$ cilliated cells and $10 \%$ Clara cells against the spread on a sample constituted of $50 \%$ ciliated cells and $50 \%$ Clara cells.

We also plan to break existing parameters into sub-models. Let us illustrate this process with an example. At the moment, we describe viral release using the parameter $g_{V}$ which describes the constant rate at which virions are released by secreting cells. In the future, this simple model of viral release could be replaced by a much more elaborate intracellular sub-model of viral assembly and release that takes account of factors such as viral strain and cell type to more accurately 
depict the dynamics. These sub-models could either be agent-based simulations or ordinary differential equations when the spatial distribution of the agents involved is not critical.

We also would like to refine the process of viral absorption, which is currently described by the parameter $k$. It has recently been shown 28 29] that susceptibility to a particular influenza strain is different depending on the cell type. For example, human influenza virions preferentially bind to sialic acid (SA)- $\alpha$ 2,6-Gal terminated saccharides found on the surface of ciliated epithelial cells of the upper respiratory tract while avian influenza H5N1 prefers (SA)- $\alpha-2,3-\mathrm{Gal}$ found on goblet cells in and around the alveoli [2829]. One easy way to take this type of heterogeneity into consideration would be to define a virion absorption rate rather than an infection rate, and consider different production rates, $g_{V}$, for each strain of virus and for each cell type. Eventually, the parameter for the absorption rate of virions, for example, could be broken into a sub-model describing the molecular processes involved in virion absorption which would explain in which way virus strains and cell receptors affect its value.

Eventually, when mechanisms such as viral absorption and release have been modified to take on the form of molecular sub-models, the ABM will be calibrated against a few different known influenza strains. This will provide pointers as to which characteristics of an influenza viral strain drive these mechanisms. Ultimately, we hope to be able to take a newly isolated influenza strain, infect our in vitro system, and then fit our ABM to the experimental results. Doing so would reveal the value of the parameters characterizing this particular strain and hence reveal the lethality and infectivity of that strain.

\section{Simulation Platform}

The model is implemented on the MASyV (for Multi-Agent System Visualization) simulation platform. MASyV facilitates the visualization of simulations without the user being required to implement a graphical user interface (GUI). The software uses a client-server architecture with the server providing I/O and supervisory services to the client ABM simulation. The MASyV package consists of a GUI server, masyv, a non-graphical command-line server for batch runs, logmasyv, and a message passing library, ma_message, containing functions to be used by the client to communicate with the server. The simulation framework is written in $\mathrm{C}$ and was developed on a Linux (Debian) system.

With the MASyV framework, a user can write a simple two-dimensional client program in $\mathrm{C}$, create the desired accompanying images for the agents with a paint program of her/his choice (e.g. GIMP), and connect the model to the GUI using the functions provided in the message passing library. The flexible GUI of MASyV, masyv, supports data logging and visualization services, and it supports the recording of simulations to a wide range of video formats, maximizing portability and the ability to share simulation results collaborators. The GUI, masyv, is built using GTK+ widgets and functions. For better graphics performance, the display screen widget, which displays the client simulation, uses GtkGLExt's 
OpenGL extension which provides an additional application programming interface (API) enabling GTK+ widgets to rapidly render scenes rapidly using OpenGL's graphics acceleration capabilities. Capture of the simulation run to a movie file requires the software Transcode 30 and the desired compression codecs be installed on the user's machine.

For non-graphical batch runs, a command-line interface, logmasyv, is also implemented. This option is designed to run multiple simulation runs (e.g. for parameter sweeps on large computer grids). This option requires only that a $\mathrm{C}$ compiler be available, and it eliminates the substantial CPU overhead cost incurred by the graphical services. Communication between the server program (either masyv or logmasyv) and the client simulation is done through a Unix domain socket stream.

MASyV is open source software distributed under the GNU General Public License (GNU GPL) and is freely available for download from SourceForge [31. It has a fixed web address, it is well maintained and documented, has an online tutorial, and comes with a "Hello World" client simulation demonstrating how to implement a new client and how to make use of the message passing library. MASyV also comes with a few example pre-programmed clients such as an ant colony laying and following pheromone trails (ma_ants) and a localized viral infection (ma_immune) which was used in [1132. Our influenza model was derived from ma_immune and is now distributed with MASyV under the name ma_virions.

\section{Conclusion}

We have described the implementation of an agent-based simulation built to reproduce the dynamics of the in vitro infection of a lung epithelial cell monolayer with an influenza A virus. At this time, model development is still in its preliminary stage, and many details remain to be elucidated. However, preliminary runs with biologically realistic parameter values have yielded reasonable results when compared with the currently available experimental data.

Recent results from the in vitro experiments revealed that large numbers of virions were being trapped by the mucus. While at $1 \mathrm{~h}$ post-harvest viral assays revealed that the experimental well contained about 4,701 \pm 180 virions, it contains a mere $635 \pm 273$ virions only $1 \mathrm{~h}$ later at $2 \mathrm{~h}$ post-harvest and $720 \pm 240$ virions at $4 \mathrm{~h}$ post-harvest. These new results suggest that trapping of the virions by the mucus and the absorption of virions by the epithelial cells upon infection plays a crucial role in controlling the rate of spread of the viral infection. In light of these new results, we plan to direct our future research towards better characterizing the role of the mucus in viral trapping and its effect on viral infectivity.

This recent development is an excellent example of just how much we still need to learn about influenza infection. It also shows that our strategy of combining in vitro and in silico tools will prove a useful tool in this quest. 


\section{References}

1. World Health Organization: Influenza. Fact Sheet 211, World Health Organization (Revised March 2003) Available online at: http://www.who.int/ mediacentre/factsheets/fs211/

2. Webster, R.G., Peiris, M., Chen, H., Guan, Y.: H5N1 outbreaks and enzootic influenza. Emerg. Infect. Dis. 12(1) (2006) 3-8

3. Tumpey, T.M., Basler, C.F., Aguilar, P.V., Zeng, H., Solórzano, A., Swayne, D.E., Cox, N.J., Katz, J.M., Taubenberger, J.K., Palese, P., García-Sastre, A.: Characterization of the reconstructed 1918 Spanish influenza pandemic virus. Science 310(5745) (2005) 77-80

4. Tumpey, T.M., García-Sastre, A., Taubenberger, J.K., Palese, P., Swayne, D.E., Pantin-Jackwood, M.J., Schultz-Cherry, S., Solórzano, A., Van Rooijen, N., Katz, J.M., Basler, C.F.: Pathogenicity of influenza viruses with genes from the 1918 pandemic virus: Functional roles of alveolar macrophages and neutrophils in limiting virus replication and mortality in mice. J Virol 79(23) (2005) 14933-14944

5. Belz, G.T., Wodarz, D., Diaz, G., Nowak, M.A., Doherty, P.C.: Compromized influenza virus-specific $\mathrm{CD} 8^{+}-\mathrm{T}$-cell memory in $\mathrm{CD} 4^{+}$-T-cell-deficient mice. J. Virol. 76(23) (2002) 12388-12393

6. Fritz, R.S., Hayden, F.G., Calfee, D.P., Cass, L.M.R., Peng, A.W., Alvord, W.G., Strober, W., Straus, S.E.: Nasal cytokine and chemokine response in experimental influenza A virus infection: Results of a placebo-controlled trial of intravenous zanamivir treatment. J. Infect. Dis. 180 (1999) 586-593

7. Kilbourne, E.D.: Influenza. Plenum Medical Book Company, New York (1987)

8. Larson, E., Dominik, J., Rowberg, A., Higbee, G.: Influenza virus population dynamics in the respiratory tract of experimentally infected mice. Infect. Immun. 13(2) (1976) 438-447

9. Bocharov, G.A., Romanyukha, A.A.: Mathematical model of antiviral immune response III. Influenza A virus infection. J. Theor. Biol. 167(4) (1994) 323-360

10. Baccam, P., Beauchemin, C., Macken, C.A., Hayden, F.G., Perelson, A.S.: Kinetics of influenza A virus infection in humans. J. Virol. 80(15) (2006)

11. Beauchemin, C., Samuel, J., Tuszynski, J.: A simple cellular automaton model for influenza A viral infections. J. Theor. Biol. 232(2) (2005) 223-234 Draft available on arXiv:q-bio.CB/0402012.

12. Celada, F., Seiden, P.E.: A computer model of cellular interactions in the immune system. Immunol. Today 13(2) (February 1992) 56-62

13. Efroni, S., Harel, D., Cohen, I.R.: Toward rigorous comprehension of biological complexity: Modeling, execution, and visualization of thymic T-cell maturation. Genome Res. 13(11) (2003) 2485-2497

14. Meier-Schellersheim, M., Mack, G.: SIMMUNE, a tool for simulating and analyzing immune system behavior. arXiv:cs.MA/9903017 (1999)

15. Polys, N.F., Bowman, D.A., North, C., Laubenbacher, R.C., Duca, K.: PathSim visualizer: An Information-Rich Virtual Environment framework for systems biology. In Brutzman, D.P., Chittaro, L., Puk, R., eds.: Proceeding of the Ninth International Conference on 3D Web Technology, Web3D 2004, Monterey, California, USA, 5-8 April 2004, ACM (2004) 7-14

16. Warrender, C.E.: CyCells. Computer Software distributed on SourceForge under the GNU GPL at: http://sourceforge.net/projects/cycells. (2005)

17. Segovia-Juarez, J.L., Ganguli, S., Kirschner, D.: Identifying control mechanisms of granuloma formation during $M$. tuberculosis infection using an agent-based model. J. Theor. Biol. 231(3) (2004) 357-376 
18. Warrender, C., Forrest, S., Koster, F.: Modeling intercellular interactions in early Mycobaterium infection. B. Math. Biol. (in press)

19. Edelstein-Keshet, L., Spiros, A.: Exploring the formation of Alzheimer's disease senile plaques in silico. J. Theor. Biol. 216(3) (2002) 301-326

20. Abbott, R.G., Forrest, S., Pienta, K.J.: Simulating the hallmarks of cancer. Artif. Life in press (2006)

21. Gerety, R., Spencer, S.L., Pienta, K.J., Forrest, S.: Modeling somatic evolution in tumorigenesis. PLoS Comput. Biol. in review (2006)

22. González-García, I., Solé, R.V., Costa, J.: Metapopulation dynamics and spatial heterogeneity in cancer. PNAS 99(20) (2002) 13085-13089

23. Maley, C.C., Forrest, S.: Exploring the relationship between neutral and selective mutations in cancer. Artif. Life 6(4) (2000) 325-345

24. Maley, C.C., Forrest, S.: Modeling the role of neutral and selective mutations in cancer. In Bedau, M.A., McCaskill, J.S., Packard, N.H., Rasmussen, S., eds.: Artificial Life VII: Proceedings of the 7th International Conference on Artificial Life, Cambridge, MA, MIT Press (2000) 395-404

25. Maley, C.C., Reid, B.J., Forrest, S.: Cancer prevention strategies that address the evolutionary dynamics of neoplastic cells: Simulating benign cell boosters and selection for chemosensitivity. Cancer Epidem. Biomar. 13(8) (2004) 1375-1384

26. Strain, M.C., Richman, D.D., Wong, J.K., Levine, H.: Spatiotemporal dynamics of HIV propagation. J. Theor. Biol. 218(1) (2002) 85-96

27. Zorzenon dos Santos, R.M., Coutinho, S.: Dynamics of HIV infection: A cellular automata approach. Phys. Rev. Lett. 87(16) (2001)

28. Shinya, K., Ebina, M., Yamada, S., Ono, M., Kasai, N., Kawaoka, Y.: Influenza virus receptors in the human airway. Nature 440(7083) (2006) 435-436

29. van Riel, D., Munster, V.J., de Wit, E., Rimmelzwaan, G.F., Fouchier, R.A., Osterhaus, A.D., Kuiken, T.: H5N1 virus attachment to lower respiratory tract. Science 312(5772) (2006) 399 Originally published in Science Express on 23 March 2006.

30. Östreich, T., Bitterberg, T., et al.: Transcode. Computer software distributed under the GNU GPL at: http://www.transcoding.org. (2001)

31. Beauchemin, C.: MASyV: A Multi-Agent System Visualization package. Computer software distributed on SourceForge under the GNU GPL at: http://masyv. sourceforge.net. (2003)

32. Beauchemin, C.: Probing the effects of the well-mixed assumption on viral infection dynamics. J. Theor. Biol. in press (2006) Draft available on arXiv:qbio.CB/0505043. 